\title{
Human gait recognition using orthogonal least square as feature selection
}

\author{
Rohilah Sahak ${ }^{1}$, Nooritawati Md Tahir ${ }^{2}$, Ahmad Ihsan Mohd Yassin ${ }^{3}$, \\ Fadhlan Hafizhelmi Kamaruzaman ${ }^{4}$ \\ ${ }^{2,3,4}$ Faculty of Electrical Engineering, Universiti Teknologi MARA, Malaysia \\ ${ }^{1}$ First City University College, Persiaran Bandar Utama, Malaysia \\ ${ }^{2}$ National Design Centre (NDC), Universiti Teknologi MARA, Malaysia
}

\begin{tabular}{|c|c|}
\hline Article Info & ABSTRACT \\
\hline Article history: & This study investigates the potential gait features that are related to human \\
\hline Received Aug 10, 2019 & $\begin{array}{l}\text { recognition using orthogonal least square (OLS). Firstly, video of } 30 \text { subjects } \\
\text { walking in oblique view was recorded using Kinect. Next, all } 20 \text { skeleton }\end{array}$ \\
\hline Revised Sep 1, 2019 & joints in 3D space were extracted and further selected using OLS. \\
\hline Accepted Oct 1, 2019 & Additionally, SVM with linear, polynomial and radial basis function (RBF) \\
\hline Keywords: & $\begin{array}{l}\text { proven to be able to identify the significant features using all three kernels of } \\
\text { SVM since all recognition accuracy attained is higher as compared to the }\end{array}$ \\
\hline Human gait recognition & original gait features. Results attained showed that the highest recognition \\
\hline Kinect & kernel, followed by 46 skeleton joint points for SVM with RBF kernel \\
\hline Orthogonal least square & namely $88.33 \%$ and accuracy of $86.33 \%$ for 38 skeleton joint points using \\
\hline Support vector machine & polynomial kernel. \\
\hline
\end{tabular}

Copyright (c) 2020 Institute of Advanced Engineering and Science. All rights reserved.

Corresponding Author:

Nooritawati Md Tahir, Faculty of Electrical Engineering, Universiti Teknologi MARA, 40450 Shah Alam, Selangor, Malaysia.

Email: nooritawati@ieee.org

\section{INTRODUCTION}

Recently, study on recognition of human gait using features extracted from Kinect sensor was extensily explored and investigated. This is because the usage of Kinect in data acquisition could simplifies process of extracting gait features since Kinect sensor automatically tracks walking human by generating all coordinate of 20 skeleton joints in the 3D space [1-3]. Furthermore, gait features that are generated from the Kinect was proven significant for human recognition purpose as reported by [4-6]. Parameter such as attire, lighting along with walking view are commonly investigated in order to develop robust recognition technique.

Initially, recognition of human gait using Kinect has been explored specifically in lateral view. For instance, nine subjects were required to walk from right to left, in front of the Kinect for eight times $[7,8]$. Then, 11 sets of static features consisting of height, length of legs, torso, both lower legs, both thighs, both upper arms and both forearms, and two sets of dynamic features; the length of step and the speed were extracted. The researchers further examined the potential gait features related to human recognition using Naïve Bayes, One Rule (1R) and C4.5 algorithm. The outcome was a high recognition accuracy of $91 \%$ using combination of four static gait features and Naïve Bayes as classifier. The four static gait features identified as significant were height, length of legs, length of torso and length of the left upper arm. Conversely, recognition of human gait in lateral view was further investigated in the study of [9-11]. In these studies, combination of dynamic and static features was found to be highly significant for human recognition as 
compared to static and dynamic features solely. Additionally, recognition of human gait was further extended to other gait views such as frontal, oblique and multi-view. Similar to lateral view, human gait could be highly recognize using fusion of static and dynamic features with classifiers such as Multi-Layer Perceptron (MLP), Support Vector Machine (SVM) [12-15] and k-Nearest Neighbor (kNN) [16], for both frontal view and multi-view [17]. Meanwhile, for oblique view, to the best of our knowledge, only one study reported on the recognition of human gait in oblique view. The researchers performed recognition of 10 subjects walking in lateral, oblique and frontal views in front of the Kinect using WEKA application along with LibSVM and One Class Classifier were employed to classify gait pattern according to its group. The results attained showed that the most significant feature was the fusion of seven static features; the length of the right and left shoulder, height, the length of the right arm and the length of the right knee and right foot and the length of the right hip. Conversely, orthogonal least square (OLS) has been used to select the most significant features in pattern recognition area as reported in [18-20]. Primarily, OLS algorithm has been utilised in system identification [21, 22] and structure of classifier model [23, 24]. Though OLS has never been used in gait recognition for feature selection, based on past researches that reported good performance of OLS as discussed in $[18,19]$, the potential of OLS as feature selection will be investigated in this study. Therefore the recognition of human gait in oblique view along with OLS as features optimization and Support Vector Machine (SVM) as classifier will be further explored and investigated.

\section{RESEARCH METHOD}

Figure 1 depicts the recognition of human gait using OLS and SVM for oblique view. Firstly, video of walking human was recorded by the Kinect during data acquisition. The recorded video was captured at 30 frames per second with resolution of 640 x 480 . Then, skeleton joints generated from the Kinect are extracted. In order to identify skeleton joints related to human gait recognition, OLS is used to select the significant skeleton joints and further classify by SVM. To evaluate the significant skeleton joints, recognition accuracy is computed based on average of 10-cross validation method.

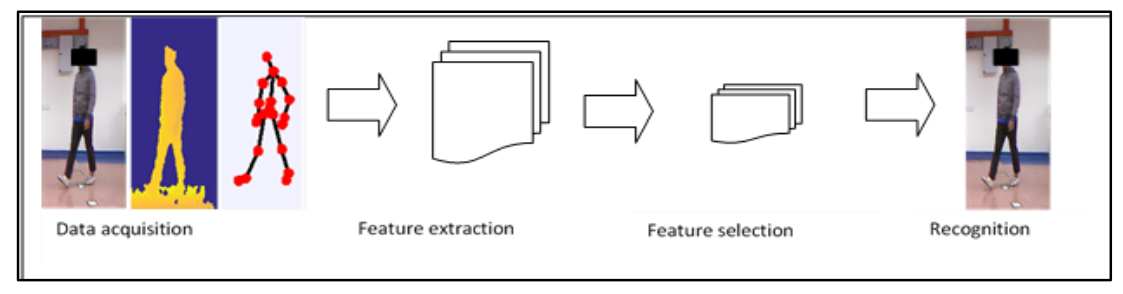

Figure 1. Recognition of human gait for oblique view

During data acquisition stage, 30 subjects were required to walk obliquely, in front of the Kinect, as shown in Figure 2. Here, layout measurement employed was based on the standard measurement of Kinect. The red area indicates the covered monitoring area and area of walking by subjects is recorded. In order to gain normal walking patterns, the participants began walking outside of the covered area. The subjects were requested to walk repeatedly for 10 times and no restriction on clothing types. In addition, the participants are required to walk naturally using their comfortable walking style and speed. Next, skeleton joints within a full gait cycle are extracted in the feature extraction stage. This stage involves five steps; removal of empty video frames, normalization of skeleton joints, detection of gait cycle, synchronization of frame number and lastly, extraction of skeleton joints. In the first step, empty video frames recorded during data acquisition are removed. Then, skeleton joints are normalized at a constant size. From observation, an inconsistency size of human skeleton attained as the participant moves toward the Kinect. Here, the size of human skeleton at a half of the trimmed video was chosen as the fix size. In addition, relative movement of skeleton joints was computed by considering head joint as the reference point. After that, a full gait cycle was detected by tracing three consecutive local minima in the vector of distance between the joint of left ankle and right ankle [4]. The distance vector was computed using Euclidean distance (1), in z-axis of the joints, and then filtered and smoothed using Savitzky-Golay moving average algorithm as:

$$
d(a, b)=\sqrt{\left(\mathrm{a}_{\mathrm{z}}-b_{z}\right)^{2}}
$$

where $\mathrm{a}$ is the joint of left ankle and $\mathrm{b}$ is the joint right ankle. 


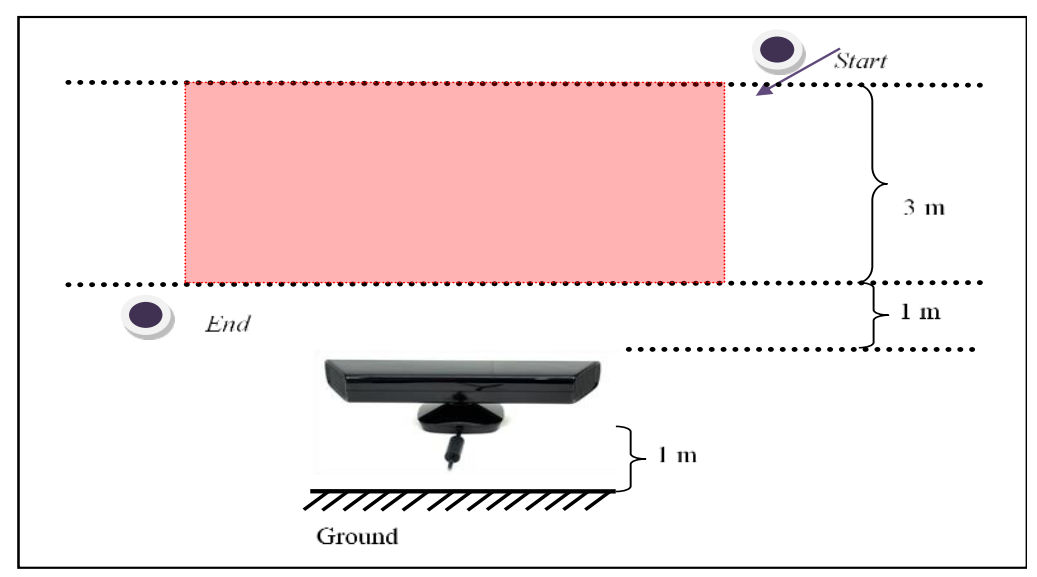

Figure 2. Recording area with its layout measurement

As consequences, one to two gait cycles with various frame numbers were attained for all walking sequences. The various frame numbers (12 to 26 frames) further can lead to intricacy in the recognition stage. Therefore, in the synchronization of frame number stage, spline interpolation was employed to standardize the frame numbers at a maximum frame number (26 frames). Lastly, in the feature extraction stage, skeleton joints in each xyz-axis; which also known as skeleton joint points, as listed in Table 1 were extracted within a full gait cycle, using (2).

$$
\text { Feature vector }=\left(j_{x_{n}} j_{y_{n}} j_{z_{n}} \times m\right) \times s
$$

where $j_{x n}=$ number of skeleton joint in x-coordinate, $j_{y n}=$ number of skeleton joint in y-coordinate, $j_{z n}=$ number of skeleton joint in $\mathrm{z}$-coordinate, $m=$ number of frame and $s=$ number of sample.

Table 1. Skeleton Joint Point with Its Label

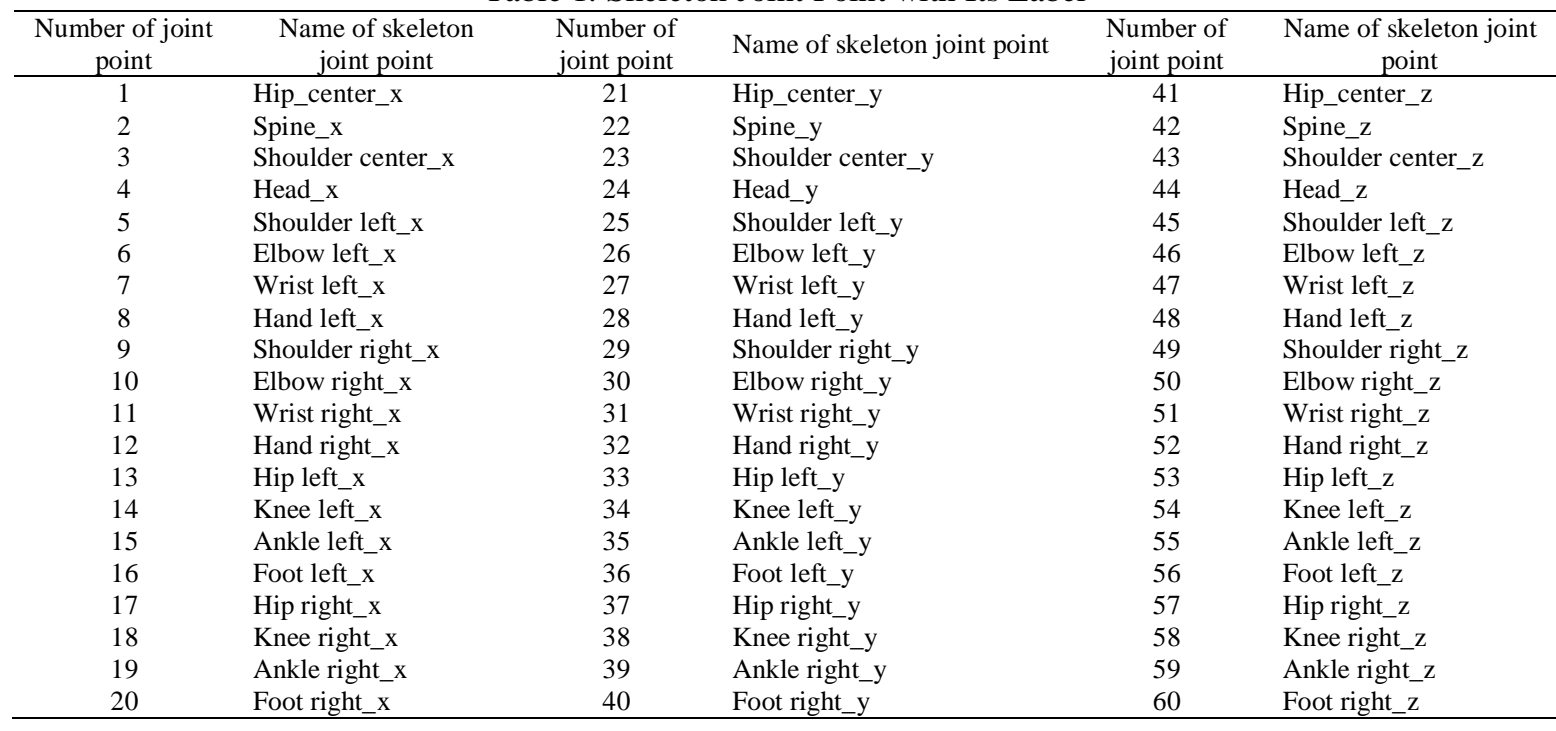

For standardization purpose, only gait features within one gait cycle for each walking sequence per participant was used for the next stage. In the feature selection stage, the OLS algorithm calculates Error Reduction Ratio (ERR) for each skeleton joint in the model. From these, the percentage reduction made by each skeleton joint with respect to the output Mean Squared Error (MSE) is derived. The significance of each skeleton joint is ranked according to its contribution in reducing error in the model. The higher the ERR value, the more significant the skeleton joint point is. In this study, the OLS is computed for $\mathrm{m}$ frames of each skeleton joint point. Hence, the input features for OLS computation was arranged for each frame as shown in (3): 


$$
f_{i k}=s \times j_{x_{n}} j_{y_{n}} j_{z_{n}}
$$

where $j_{x n}=$ number of skeleton joint in x-coordinate, $j_{y n}=$ number of skeleton joint in y-coordinate, $j_{z n}=$ number of skeleton joint in z-coordinate, $s=$ number of sample.

In the OLS computation, the expected output $y_{k}$ was computed as follows:

$$
y_{k}=\sum_{i=1}^{m} f_{i k} \theta_{i}
$$

where, $m$ is the number of frame, $f_{i k}$ is the input features; $\theta_{i}$ is the solution to the linear least squares problem for $i=1, \ldots, m$. With the use of Householder-based $Q R$ decomposition, the input features, $f_{i k}$ is transformed into an auxiliary model as shown in (5).

$$
y_{k}=\sum_{i=1}^{m} w_{i k} g_{i}
$$

where, $w_{i k}$ is orthogonal to one another and $g_{i}$ are constant coefficients.

The estimates of the coefficients $g_{i}$ are given by:

$$
\widehat{g}_{l}=\frac{\sum_{\mathrm{k}=1}^{\mathrm{N}} \mathrm{w}_{\mathrm{ik}} \mathrm{y}_{\mathrm{k}}}{\sum_{\mathrm{k}=1}^{\mathrm{N}} \mathrm{w}^{2}{ }_{\mathrm{ik}}}
$$

and the error expression for ERR, $\operatorname{err}_{i}$ computed as:

$$
\operatorname{err}_{i}=\frac{{\widehat{g_{l}}}^{2} \sum_{k=1}^{N} w^{2}{ }_{i k}}{\sum_{k=1}^{N} y_{k}^{2}}
$$

then, average err, ERR is computed as:

$$
\mathrm{ERR}=\frac{e r r_{i}}{m}
$$

The skeleton joint point with the highest ERR was arranged at the top and the lowest ERR was placed at the bottom. Further, the input feature was arranged using (2) with numbers of arranged skeleton joint points, starting from 2 to 60 skeleton joint points with incremental of 2 .

As a result, various numbers of feature set were obtained. In the recognition stage, SVM with linear, polynomial and Radial Basis Function (RBF) kernel were employed as the classifiers. Upon several experimental analysis, regularization parameter $(\mathrm{C})$ for linear kernel was significant to vary at $0.001,0.01$, $0.1,1$ and 10 , for polynomial and RBF kernel at 0.00001 to 0.01 with 1 decade increments and 10 to 100 with 10 increments, respectively. In addition, polynomial order (d) of polynomial kernel was experimented at 2 and 3 and sigma $(\sigma)$ for RBF kernel varied from 10 to 200. For the purpose of the evaluation of recognition performance and parameter selection of the classifier model [22], the 10-fold cross validation [25, 26] was used.

\section{RESULTS AND ANALYSIS}

In this section, the results attained based on the proposed methodology will be elaborated and discussed. As tabulated in Table 2, it can be observed that the rank of skeleton joint points after the implementation of OLS. Obviously, the joint of hip center in $\mathrm{x}$-axis dominates the first rank as it attained the highest ERR. Furthermore, it can be seen that most of skeleton joints in $\mathrm{x}$-axis placed at the first 30 rank, followed by skeleton joints in y-axis and z-axis, respectively.

Figure 3 shows the recognition accuracy attained at the optimal SVM model. High recognition accuracy attained at 48 skeleton joint points for SVM with linear kernel (90.67\%), 44 skeleton joint points for SVM with RBF kernel (88.33\%) and 38 skeleton joint points for SVM with polynomial kernel (86.33\%). In order to verify the effectiveness of OLS as feature selection, recognition accuracy for features without OLS was computed too.

Figure 4 depicts the comparison of recognition accuracy between the original features and OLS features. As can be observed, recognition accuracy are enhanced using features selected by OLS, for all SVM kernels category. 


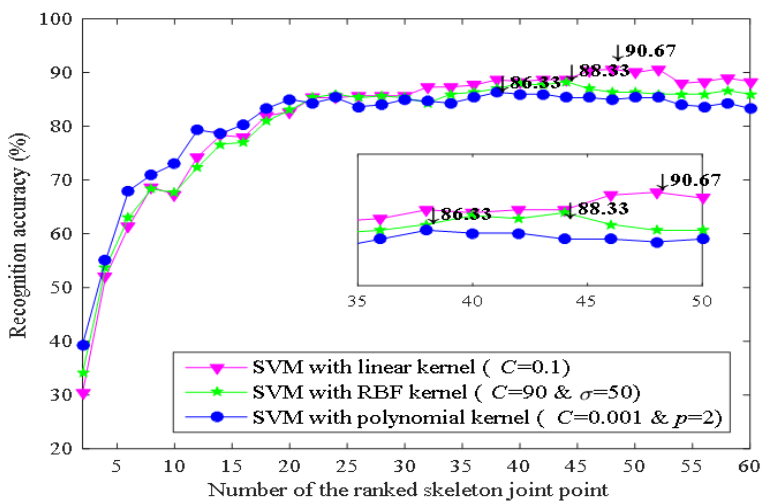

Figure 3. Recognition accuracy for various numbers of skeleton joint points accordance to OLS rank

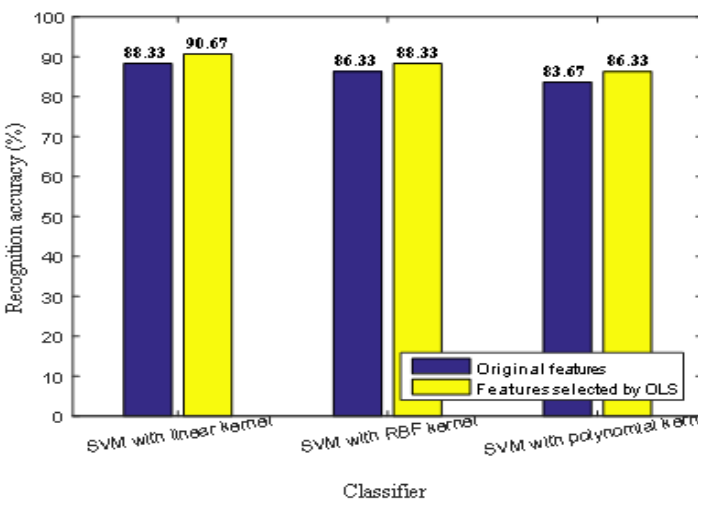

Figure 4. Recognition accuracy attained using original features and features selected by OLS for SVM

Table 2. Rank of Skeleton Joint Points after OLS Implementation

\begin{tabular}{|c|c|c|c|c|c|}
\hline Joint label & Joint name & ERR & Joint label & Joint name & ERR \\
\hline 1 & Hip_center_x & 0.699097276 & 55 & Ankle left_z & 0.000636189 \\
\hline 21 & Hip_center_y & 0.085875078 & 26 & Elbow left_y & 0.000621815 \\
\hline 4 & Head_x & 0.042695157 & 31 & Wrist right_y & 0.000621299 \\
\hline 3 & Shoulder center_x & 0.019806264 & 57 & Hip right_z & 0.000615943 \\
\hline 5 & Shoulder left_x & 0.015747802 & 29 & Shoulder right_y & 0.000592885 \\
\hline 10 & Elbow right_x & 0.014847255 & 38 & Knee right_y & 0.000588331 \\
\hline 23 & Shoulder center_y & 0.013521678 & 43 & Shoulder center_z & 0.000537843 \\
\hline 9 & Shoulder right_x & 0.004801643 & 60 & Foot right_z & 0.000521329 \\
\hline 17 & Hip right_x & 0.003904192 & 48 & Hand left_z & 0.000487427 \\
\hline 13 & Hip left_x & 0.003405773 & 47 & Wrist left_z & 0.000480826 \\
\hline 8 & Hand left_x & 0.003336582 & 28 & Hand left_y & 0.000467799 \\
\hline 14 & Knee left_x & 0.003330543 & 37 & Hip right_y & 0.000453269 \\
\hline 11 & Wrist right_x & 0.003307623 & 46 & Elbow left_z & 0.000438526 \\
\hline 7 & Wrist left_x & 0.003139608 & 41 & Hip_center_z & 0.000421997 \\
\hline 27 & Wrist left_y & 0.002716615 & 45 & Shoulder left_z & 0.000415265 \\
\hline 2 & Spine_x & 0.002675057 & 51 & Wrist right_z & 0.000407073 \\
\hline 22 & Spine_y & 0.002644155 & 53 & Hip left_z & 0.000402531 \\
\hline 6 & Elbow left_x & 0.002040701 & 42 & Spine_z & 0.000383909 \\
\hline 34 & Knee left_y & 0.001630870 & 36 & Foot left_y & 0.000355970 \\
\hline 12 & Hand right_x & 0.001394476 & 50 & Elbow right_z & 0.000353017 \\
\hline 19 & Ankle right_x & 0.001333185 & 54 & Knee left_z & 0.000348224 \\
\hline 16 & Foot left_x & 0.001321263 & 49 & Shoulder right_z & 0.000346255 \\
\hline 15 & Ankle left_x & 0.001209764 & 58 & Knee right_z & 0.000319731 \\
\hline 35 & Ankle left_y & 0.000919835 & 18 & Knee right_x & 0.000296275 \\
\hline 20 & Foot right_x & 0.000909513 & 32 & Hand right_y & 0.000266994 \\
\hline 25 & Shoulder left_y & 0.000821467 & 59 & Ankle right_z & 0.000239201 \\
\hline 24 & Head_y & 0.000785205 & 44 & Head_z & 0.000220702 \\
\hline 40 & Foot right_y & 0.000736709 & 56 & Foot left_z & 0.000208776 \\
\hline 33 & Hip left_y & 0.000674316 & 39 & Ankle right_y & 0.000201806 \\
\hline 30 & Elbow right_y & 0.000654517 & 52 & Hand right_z & 0.000198280 \\
\hline
\end{tabular}

\section{CONCLUSION}

In conclusion, recognition of human gait in oblique view using features selected by the optimized OLS and SVM has been discussed in this study. The results showed that the combination of OLS and SVM with linear kernel contributed to the highest recognition rate namely $90.67 \%$ with 48 skeleton joint points as compared to the other two kernels. Future work includes investigating the human gait recognition using other feature selection method namely linear discriminant analysis and other classifiers specifically Naive Bayesian classifier and Deep Learning Neural Network.

\section{ACKNOWLEDGEMENTS}

The authors would like to thank Ministry of Education (MOE) Malaysia for the grant awarded Niche Research Grant Scheme (NRGS) No: 600-RMI/NRGS 5/3 (8/2013), Institute of Research Management and Innovation (IRMI) Grant No: 600-RMI/DANA 5/3/PSI (195/2013) and IRMI Premier 
Laboratory, Human Motion and Gait Analysis UiTM, Shah Alam, Selangor, Malaysia for the support given to conduct this research.

\section{REFERENCES}

[1] Wang, W.-J., Chang, J.-W., Haung, S.-F., \& Wang, R.-J. (2016). "Human Posture Recognition Based on Images Captured by the Kinect Sensor." International Journal of Advanced Robotic Systems. https://doi.org/10.5772/62163.

[2] Tsai Z-R, Robust Kinect-based guidance and positioning of a multidirectional robot by Log-ab recognition, Expert Systems with Applications, 41(4), 1271-1282, 2014.

[3] Sakurai, K., Yan, M., Tanno, K., \& Tamura, H. (2017). "Gaze Estimation Method Using Analysis of Electrooculogram Signals and Kinect Sensor." Computational Intelligence and Neuroscience, 2017, 2074752.

[4] Ťupa, O., Procházka, A., Vyšata, O., Schätz, M., Mareš, J., Vališ, M., \& Mař́ik, V. (2015). "Motion tracking and gait feature estimation for recognising Parkinson's disease using MS Kinect." Biomedical Engineering Online, 14, 97. doi:10.1186/s12938-015-0092-7

[5] Han J,Shao L, Xu D and Shotton J, "Enhanced Computer Vision With Microsoft Kinect Sensor: A Review," IEEE Transactions on Cybernetics, 43(5), 1318 - 1334, 2013.

[6] Henry, P., Krainin, M., Herbst, E., Ren, X., \& Fox, D. (2012). "RGB-D mapping: Using Kinect-style depth cameras for dense 3D modeling of indoor environments." The International Journal of Robotics Research, 31(5), 647-663. https://doi.org/10.1177/0278364911434148

[7] Hernández J-J, Lópeza A, L Quintanilla, O José, L López, R Francisco, J Rangel, B Mario, A Ibarra, M Dora, L Almanza, "Detecting objects using color and depth segmentation with Kinect sensor", Procedia Technology, Vol. 3, 196-204, 2012.

[8] Nandy, A., \& Chakraborty, P. (2015). "A new Paradigm of Human Gait Analysis with Kinect." In Eighth International Conference on Contemporary Computing (IC3) (pp. 443-448). Noida.

[9] Ball, A., Rye, D., Ramos, F., \& Velonaki, M. (2012). "Unsupervised clustering of people from "skeleton" data." In 7th ACM/IEEE International Conference on Human-Robot Interaction (HRI) (pp. 225-226). Boston MA. http://doi.org/10.1145/2157689.2157767.

[10] Dikovski, B., Madjarov, G., \& Gjorgjevikj, D. (2014). "Evaluation of different feature sets for gait recognition using skeletal data from Kinect." 2014 37th International Convention on Information and Communication Technology, Electronics and Microelectronics (MIPRO), 1304-1308. http://doi.org/10.1109/MIPRO.2014.6859769.

[11] Soriano, M., Araullo, A., \& Saloma, C. (2004). Curve spreads - a biometric from front-view gait video. Pattern Recognition Letters, 25, 1595-1602. http://doi.org/10.1016/j.patrec.2004.05.017.

[12] M. Hamiane \& F. Saeed, Classification of MRI Brain Images for Computer-Assisted Diagnosis, International Journal of Electrical and Computer Engineering (IJECE) 7(5), 2555-2564, 2017.

[13] B.Richhariya \& M.Tanveer, EEG signal classification using universum support vector machine, Expert Systems with Applications, 106 (1), 169-182, 2018.

[14] N. Aji Bhaskar, "Performance Analysis of Support Vector Machine and Neural Networks in Detection of Myocardial Infarction" Procedia Computer Science, 46(1), 20-30, 2015.

[15] S.H. Kim \& J. Choi, "An SVM-based high-quality article classifier for systematic reviews," Journal of Biomedical Informatics, 47 (1), 153-159, 2014.

[16] - S. Zhang, X. Li, M. Zong, X. Zhu, and R. Wang, "Efficient kNN Classification With Different Numbers of Nearest Neighbors," IEEE Transactions on Neural Networks and Learning Systems, 29(5), 2018.

[17] Kang, Z., Deng, M., \& Wang, C. (2017). "Frontal-View Human Gait Recognition Based on Kinect Features and Deterministic Learning." In 36th Chinese Control Conference (pp. 10834-10839).

[18] M. M. G. Macedo, W. V. N. Guimarães, M. Z. Galon, C. K. Takimura, P. A. Lemos, and M. Antonio, "Computerized Medical Imaging and Graphics A bifurcation identifier for IV-OCT using orthogonal least squares and supervised machine learning," Computerized Medical Imaging and Graphics, 46 (1), 237-248, 2015.

[19] M.S. Peprah, I.O. Mensah, Performance evaluation of the Ordinary Least Square (OLS) and Total Least Square (TLS) in adjusting field data: an empirical study on a DGPS data, South African Journal of Geomatics, 6(1), pp. 73-89, 2017.

[20] R. Ahmad and H. Jamaluddin, "Orthogonal least square algorithm and its application for modelling suspension system,” Journal of Technology, 34 (1), 71-83, 2007.

[21] E. M. A. M. Mendes and S. A. Billings, "An Alternative Solution to the Model Structure Selection Problem," IEEE Transactionson Systems, Man and Cybernatics, 31(6), 597-608, 2001.

[22] Cawley, G. C., \& Talbot, N. L. C. (2010). "On Over-fitting in Model Selection and Subsequent Selection Bias in Performance Evaluation." Journal of Machine Learning Research, 11, 2079-2107.

[23] S. Chen, X. Hong, C. J. Harris, and L. Hanzo, "Fully complex-valued radial basis function networks : Orthogonal least squares regression and classification," Neurocomputing Journal Elsevier 71(16), 3421-3433, 2008.

[24] G. Huang, S. Song, and C. Wu, "Orthogonal Least Squares Algorithm for Training Cascade Neural Networks," IEEE Transactions on Circuits and Systems 59 (11) 2629-2637, 2012.

[25] Wong, T., \& Yang, N. (2017). Dependency Analysis of Accuracy Estimates in k-Fold Cross Validation. IEEE Transactions on Knowledge and Data Engineering, 29(11), 2417-2427.

[26] Lameiro, C., \& Schreier, P. J. (2016). Cross-validation techniques for determining the number of correlated components between two data sets when the number of samples is very small in 50th Asilomar Conference on Signals, Systems and Computers (pp. 601-605). Pacific Grove. 


\section{BIOGRAPHIES OF AUTHORS}
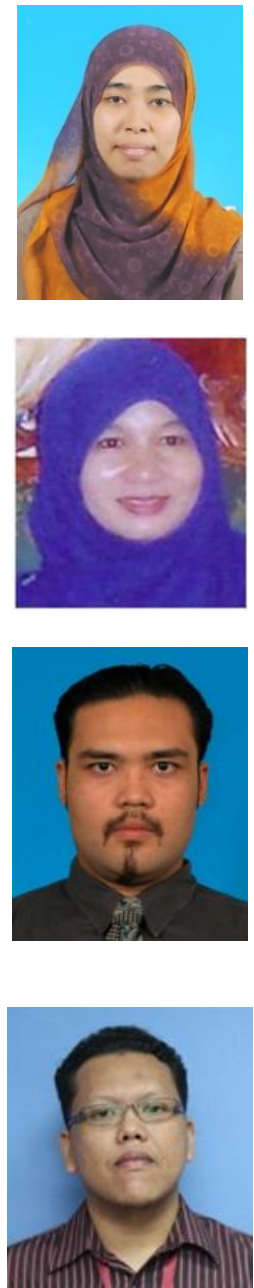

Rohilah Sahak obtained her PhD in Electrical Engineering in 2018, Master in Electrical Engineering in 2012 from Universiti Teknologi MARA (UiTM) Shah Alam, Selangor and the Bachelor of Engineering in Electronics in 2007 from University Selangor (UniSEL), Selangor. Currently, she is working as lecturer at First City University College, Petaling Jaya, Selangor. Her research interests is in the area of signal and image processing, biomedical engineering as well as pattern recognition.

Nooritawati Md Tahir received her PhD in Electrical, Electronic \& System Engineering from Universiti Kebangsaan Malaysia. She is currently a Professor at the Faculty of Electrical Engineering, Universiti Teknologi MARA, Malaysia. Her research interests include pattern recognition, computational intelligence and artificial intelligence. She is the Chair of Industrial Electronis/Industrial Application (IE/IA), Malaysia Chapter since 2016 and a registered Chartered Engineer (C Eng) with the Chartered Engineer UK.

Dr. Ir. Ahmad Ihsan Mohd Yassin graduated with Phd and MSc in Electrical Engineering from UiTM in 2014 and 2008, respectively. Currently, he is serving as a Senior Lecturer in the Centre of Computer Engineering Studies, Faculty of Electrical Engineering, and Associate Fellow in Microwave Research Institute (MRI) and Malaysia Institute of Transport (MITRANS).

His research interests are in System Identification, Artificial Intelligence (Fuzzy Logic, Neural Networks and Deep Learning), and Stochastic Optimization Methods. Dr Ir Ihsan is also registered as a Professional Engineer (PEng) with Board of Engineers Malaysia and Chartered Engineer (CEng) registered with the Institution of Engineering and Technology (IET), UK.

Fadhlan Hafizhelmi Kamaru Zaman received the B.Sc (Hons.) and P.hD. degrees from International Islamic University Malaysia in 2008 and 2015, respectively. He is currently a Senior Lecturer at Department of Computer Engineering, University of Technology MARA, Malaysia. His research interests are in surveillance system, pattern recognition, signal and image processing, artificial intelligence and computer vision. Fadhlan is also a member of IEEE, Malaysian Board of Technologist (MBOT), and a Chartered Engineer from the Institution of Engineering and Technology, UK. 